\title{
EFFECT OF RESONANCE BROADENING ON THE EVOLUTION OF THE EDGE OF A TURBULENT SPECTRUM
}

A. H, KRITZ, N, J, FISCH, AND $C, F, F$, KARNEY

\section{MASTER}

\section{PLASMA PHYSICS LABORATORY}

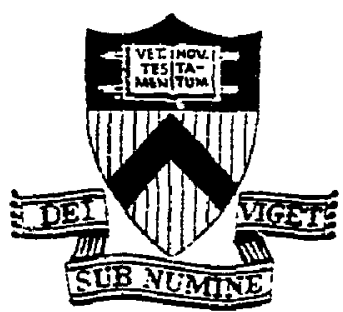

\section{PRINCETON UNIVERSITY PRINCETON, NEW JERSEY}

This work was supported by the i..5. Desartinen: of Energy

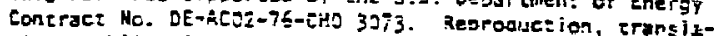
tion, publicatior, use ane cisousal. in whele or in part. by or for the Inized states goverzment is permited. 
Effect of Resonance Broadening on the Evolution of the Edge of a Turbulent Spectrum

\author{
Arnold H. Kritz \\ Plasma Physics Laboratory, Princeton University \\ Princeton, NJ 08544 \\ and \\ Hunter College of the City University of New York \\ New York, NY 10021 \\ Kathaniel J. Fisch and Charles F. F. Karney \\ Plasma Physics Laboratory, Princeton University \\ Princeton, NJ 08544
}

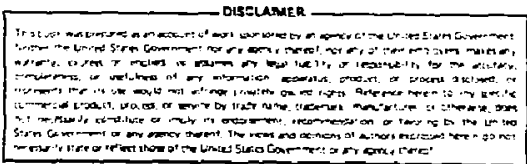


ABSTRACT

The extent to which nonlinear wave-particle resonance broadening results In a narrowing of an incldent lower-hybrid wave spectrum is investigated. This narrowing is of concern because it could make control of lower-hybrid heating difficult. We numerically stow, however, that relatively uniform spatial power deposition occurs if resonance broadening effects are treated consistenty on both the wave spectrum and the particle distribution. A more naive approach, Including only the effects on the evolution of the wave spectrum, would incorrectly predict an unfavorable powe: depositian profile. 


\section{INTRODUCTION}

The injection of lower hybrid waves into a tokamak plasma has been suggested as a means of heating the plasma to ignition ${ }^{1}$ or as a means of driving the torodial plasma current. ${ }^{2}$ The success of both schemes is based on the absorbtion of the rfopower by plasma electrons and depends, in part. on the expectation that the spatial deposition of the $r f$-power can be controlled, In particular, for heating electrons or generating current, it is likely that the mogt desirable deposition of lower hybrid $\mathrm{rf}$-power would be near the plasma center where the temperature and density profiles are relatively flat. The goal of the present study is to assess whether the nonlinear effect of resonance broadening ${ }^{3}$ interferes with the control that we hope to retain over the power deposition.

To facilitate our assessment of resonance broadening effects, we must model the interaction of the waves with the plasma in a manner that is realistic, yet isolates the effects of resonance broadening from other effects. Specifically, we shall model the plasma as a homogeneous slab. We are motivated by the following qualitative description of the spatial deposition of the rf-power: We imagine that the paralleI (to the magnetic field) wave phase velocity is chosen large compared to the electron thermal velocity near the plasma periphery, but only three to four times the thermal velocity at some hotter interior point. Thus, exponentially few electrons are resonant with the wave near the cool periphery so that the wave propagates relatively undamped until it reaches an interior point. There the plasma is warmer sc that a substantial number of resonant electrons are presnt. The wave power is absorbed between this interior point and the plasma center (the magnetic axis). To the extent that the interior point in 
question and the plasma cerier are close together, the plasma can be modeled as nearly homogeneous. Although the model may not be entirely accurate, it does isolate for us the resonance broadining effects from the effects of temperature and density gradients. The other simplification that we employ in modeling the power deposition is that the waves are incident upon an infinite half-space, rather than converging upon the magnetic axis. This modeling isolates for us the resonance broadening effects from the effects of cylindrical geometry.

In order to understand the role of resonance broadening with regard to spatial deposition of $\mathrm{rf}$-power, it is important to distinguish low-power injection from high-power injection. By low-power injection we mean that the $r f-p o w e r$ is so weak, or the plasma collisionality is so strong, that the electron velocity distribution remains nearly Maxwellian. (It is understood that the relative plasma collisionality depends, in part, on the wave phase velocity. ${ }^{2}$ ) In this limit the damping coefficient for the waves is independent of their power. In a uniform plasma, the transmitted wave power would decay exponentially with distance into the plasma since the damping coefficient is proportional to the velocity derivative of the distribution function. That is $d D / d t=Y D$ where $D$ is a measure of the incident $r f$-power and where $\gamma \alpha \partial F / \partial v$ is essentially independent of $D$.

In the opposite limit of high-power rf-waves, the collisionality of the plasma is too small to restore the Maxwellian electron velocity distribution. Instead, a plateau forms on the tail of the parallel velocity distribution at the velocity correspnding to the wave phase velocity. Thus, in the high power limit, the slope of the distribution function and, consequently, the damping coefficient for the waves is invorsely proportional to the wave power so that it is the rate of power deposition, and not the damping coefficient, 
that is independent of the wave power, $1 . e ., d D / d t \alpha(1 / D) D$. Therefore, the transmitted wave power hould only decay linearly with distance into a uniform plasma. However, even when high power lower hybrid waves are injected into the plasma, there are some components in the velocity-space spectrum, in particular those near the edge of the spectrum, whose decay are governed by the low power limit. That there exists some spectral components in the low-power regime is assured if the spectrum is continuous. Thus, we expect that under high-power injection, the edge of the wave spectrum must sharpen because the Intensity of the components near the spectrum edge are in the low-power regime and experience exponential decay relative to the lirear decay of the components in the central portion of the spectrum. Associated with this sharpening of the spectrum, there would be a narrowing of the spectrum because of the faster decay of the edge of the spectrum. However, narrowing can occur independently, i.e., even when the spectrum is flat. The narrowing of the spectrum can occur by virtue of resonance broadening, whish assures, among other things, that the damping rate of the spectral corponents undergoes a continous transition between low- and high-power limits. In particular, the spectral components within a resonance broadening width ${ }^{3}$ of the spectrum edge would experience a damping rate larger than the components in the center of the spectrum. Physically this corresponds to these spectral components exchanging energy with electrons that have velocities outside the range of the wave phase velocities associated with the spectrum - i.e., with electrons for which the velocity distribution function presumably has a larger slope. If the resonance broadening width is broader than the characteristic width associated with the!edge of the wave spectrum, then it can be imagined how the spectrum can narrow independently of the sharpening of the edge. 
Even a small narrowing of the wave spectrum may be of great importsace because it affects the value of $v_{1}$, the lowest parallel phase velocity in the wave spectrum. The number of resonant electrons in the steady state scales as $N_{r} \sim \exp \left(-v_{1} / 2 v_{t e}{ }^{2}\right)$ where $v_{t e}$ is the electron thermal velocity and is a function of distance into the plasma. The sensitive dependence of $f_{r}$ on $v_{1}$ is reflected in the power deposition. A small narrowing of the spectrum implies that the power in the edge spectral components is quickly (exponentially) absorbed by the plasma. As a consequence, $v_{1}$ becomes a function of distance into the plasma. Associated with the increase in $v_{1}$, the plasma becomes transparent to the central spectral components. Thus, the narrowing of the spectrum would give a very unwelecome profile of power deposition.

The concern of this study is whether, due to resonance broadening, the narrowing of the wave spectrum occurs in the hlgh-power limit. This concern is particularly appropriate in the case of current generation in tokamak reactors where one starts with a narrow spectrum incident on the plasma. We will, in fact, show that this narrowing does not occur when the effects of resonance broadening are included in a proper and self-consistent manner in the evolution of both the wave spectrum and the electron velocity distribution. The concern, although eventually discounted, is nevertheless genuine and could not have been alleviated without a numerical calculation. We will show how a more naive formulation of the problem, including the resonance broadening effects on the evolution of the wave spectrim only, does lead to a narrowing of the spectrum and an unfavorable deposition profile in the manner described above.

The paper is organized as follows: In Sec. II we write down.the basic equations and pose the concern raised in this section in a more quantitative 
manner. The derivation of these equations is found in Appendix A. In Sec. III we present the numerical solution of the resunance broadening equations and we demonstrate the importance of self-consistency in formulating these equations, We conciude with a discussion of our resuits in Sec. IV. 


\section{BASIC EQUATIONS}

The evolution of the parallel electron velocity distribution, $F$, may be described by a Fokker-Planck equation (written in parailel velocity only) with an added quasilinear diffusion term due to the waves, i.e.,

$$
\frac{\partial F}{\partial \tau}=\frac{\partial}{\partial w}\langle D(w, \xi, \tau)\rangle \frac{\partial F}{\partial w}+\left(\frac{\partial F}{\partial \tau}\right)_{c},
$$

where the quasilinear diffusion coefficient $D$ depends on $F$ [see Eqs. (4) and (5) below]. All quantities are written using the normalizationg introduced in Appendix A. Thus, $\tau$ is time in units of inverse collision frequency, $w$ is p rallel electron velocity in units of the electron thermal speed and $\xi$, the normalized spatial variable, is a measure of distance into the plasma. We use the average operator $\langle>$ defined by

$$
\langle G(w)\rangle=\frac{1}{2 \delta} \int_{W-\delta}^{W+\delta} G\left(w^{\prime}\right) d w^{\prime}
$$

where $\delta$ is a given resonance broadening width and $G(w)$ is an arbitrary function, which is averaged over this width. The collision operator is given by

$$
\left(\frac{\partial F}{\partial \tau}\right)=\frac{\partial}{\partial W} \frac{1}{w^{3}} \frac{\partial F}{\partial W}+\frac{1}{W^{2}} F
$$

This operator is linearized and written in the high-velocity limit. Also, a Maxwellian distribution has been assumed for the perpendicular velocity direction. The justification for using this collision operator in the present problen follows the arguments offered in References 2 and 4 . The wave diffusion coefficient, $D$, which is proportional to the incident. wave 
power (see Appendix A), evolves according to the following equation:

$$
\frac{\partial D}{\partial \tau}+V_{g} \frac{\partial D}{\partial \xi}=\gamma(w, \xi, \tau) D
$$

where $V_{B}=W$ and $\xi$ are the dimensionless radial group velocity and spatial coordinate as shown in Appendix A. The wave damping rate, $\gamma$, is given by

$$
\gamma=A w|w| \frac{\partial\langle F\rangle}{\partial w}
$$

where the constant $A$ is defined in Eq. (A12). Below we discuss some of the properties of Eqs. (1)-(5).

Note that if collisions are neglected, i.e., $(2 F / \partial \tau)_{c}=0$, Eqs. (1)-(5) conserve energy only if the resonance broadening width, $\varepsilon$, is equal to zero. (Note that momentum is conserved even for $\delta \neq 0$. ) When $\delta$ is not equal to zero, conservation of energy is maintained only if we do not use a function to approximate the resonance broadening operator derived in Reference 3 . However, the equations would then assume a far more complicated form. Alternatively, we could force energy conservation in the manner described in Reference 5, but we do not consider this necessary for the present application. Energy is nearly conserved if $\delta$ is small. Horeover, in the presence of collisions, the energy and momentum of the resonant electrons and waves are not separately conserved. Thus, the solution for $F$ in the presence of collisions is not sensitive to small discrepancies in the separate balance of energy and momentum between the resonant electrons and the waves. 6 on the other hand, Eqs. (1)-(5) do advantageously preserve the non-negative nature of both $F$ and $D$, no matter how $\delta$ is chosen. Thus, for the present application, where the energy that the resonant electrons gain from the waves is to be balanced against the energy they lose by colliding with nonresonant 
electrons, our approach of introducing the phenomena of resonance broaden:ng in an approximate but simple manner (with desirable mathematical properties) retains the essential physies.

Our interest lies in obtaining the steady-state solution of Eqs. (1)-(5). Taking $\partial F / \partial \tau=0$ in Eq. (1), we immediately find

$$
F=\exp \left\{-\int_{C}^{w} \frac{w}{1+\langle D\rangle w^{3}} d w\right\}
$$

where $c(\xi)$ is determined by the condition that the electron density [1.e.. $F(w)$ integrated over the parallel velocity $w$ ] remains a (given) function of $\xi$ only. Note that $F(w)$ is Maxwellian where $\langle D\rangle$ vanishes and is flat where $(D\rangle$ is large. Furthermore, note that the height of the plateau where $F$ is flat is exponentially sensitive to the value of the slowest phase velocity in the wave spectrum.

The power carried by the transmitted wave may now be determined using Eqs. (4) $-(6)$ with $\partial D / \partial \tau=0$. The concern regarding resonance broadening, expressed in Sec. I, stems from Eq. (4). Near the spectrum edge, the damping rate tends to be much larger when the averaged $F$ is employed instead of the unaveraged $F$. The effect of this larger damping rate is that the edge spectral components are lost faster than the central components. However, we shall see below that this effect is mitigated when the $D$ used in Eq. (6) is averaged cyer the resonance broadening width, as opposed to not being averaged. The reduction of the resonance broadening effect occurs because the particle distribution becomes flattened somewhat even outside the range of the spectrun phase velocities when $\langle D\rangle$ is used.

In the next section, we will present numerical solutions of Eqs. $(4)-(6)$. When the resonance broadening effects are included in both 
evolution equations, $1 . e .$, for the waves and for the electrons, we refer to the solutions as solutions of the consistent set of equat,ions. What we refer to as solutions of the inconsistent set of equations are solutions of Eqs. (4)-(6) but with the uraveraged value of D (naively) employed in Eq. (6) instead of the resonance broadened value $\langle D\rangle$. It will be shown that it is only the inconsistent set of equations that exhibits the severe narrowing of the spectrum and the consequent unfavorable power deposition profile. 
III. NUMERICAL SOLUTION

In order to show the effect of resonance broadening on the propagation nf lower hybrid waves and deposition of their power, we choose a typical set of parameters and display various aspects of the steady state solutions of Eqs. (4)-(6) with (a) no resonance broadening, i.e., $\delta=0$; (b) consistent resonance brvadening; and (c) inconsistent resonance broadening, i.e., $\delta \neq 0$ in Eq. (5) and $\delta=0$ in Eq. (6).

He chose $D(w, \xi=0)=30 / w$ for $3.6<\mathrm{H}<6.0$ and zero outside this range of parallel phase velocity. This is representative of lower hybrid waves with frequency of $1.2 \mathrm{GHz}$ and fower levels of $1 \mathrm{MW}$ propagating in a plasma with electron density $2.5 \times 10^{13} \mathrm{~cm}^{-3}$ and electron temperature $\mathrm{T}_{\mathrm{e}}=2.5 \mathrm{keV}$. The resonance broadening width is taken to be constant equal to $0.1 \mathrm{v}_{\text {te }}$, i.e., $\delta=0.1$. Taking $\delta$ to be a constant and making no attempt to relate it back to $\left(D / k_{y}\right)^{1 / 3}$ is not strictly correct but suffices here in our examination of the nature of the influence that resonance broaciening has on the deposition of power.

The variation of the spectral power density as a function of $\xi$ is shown in Fig. 1. It is seen that with no resonance broadening, curve (a), and consistent resonance broadening, curve (b), the decay of the spectrum is nearly linear. The decay in case (b) is somewhat faster than in case (a). However, with inconsistent resonance broadening, curve (c), a rapid initial decay of the spectrum is followed by a much slower decay of the spectrum. Case (c) is illustrative of the effect that we had feared before doing the problem self-consistently.

The results shown in Fig. 1 are illustrated more succinctly in Figs. 2 and 3. In Fig. 2 we plot as a function of $\xi$ the fraction of the total power 
carried by the lower hybrid waves and in Fig. 3, the rate of power deposition. Note in Fig. 3 the uniform deposition of power in case (a) and (b) compared with the non-uniform deposition in case (c). The origin of this behavior car be seen in Fig. 4 where we plot the location of the inner (low velocity) edge of the spectrum as a function of $\xi$ for the three cases. The location of this edge is defined by the iesser of the two solutions to

$$
D\left(w_{1}\right)=0.5 \max _{w}[D(w)]
$$

Whereas, in cases (a) and (b) the edge is nearly stationary until a major fraction of the $r$ fi-energy has been deposited, in case (c) the edge moves to larger velocities with increasing 5 (propagation into the plasma) reducing the lumber of resonant particles and hence the damping rate. 
IV. CONCLUSIONS

We have examined the extent to which nonlinear resonarce broadenting affects a lower hybrid rf-spectrum. While one might expect resonance broadening on the waves to dramatically narrow the spectrum, it is shown that this effect is counteracted by resonance broadening on the particles, which extends the plateau In the parallel veloctty distribution a resonance broadening width into the non-resonance region. Thus, when resonance broadening is treated consistently, the rf-power spectrum does not narrow signiflcantly and the uniform spatial deposition of the rf-power is retained.

ACKNOWLEDGEMENTS

This work was supported by the Unfted States Department of Energy under Contract No. DE-AC02-76-CH03073.

One of the authors (AHK) was also supported in part by a grant from the PSC-BHE Research Award Program of the City University of New York. 
APPENDIX A

It can be shown that when the plasma is subject to the incident rf-electrostatic waves, the one-particle elestron velocity distribution function satisfies the equation

$$
\frac{\partial}{\partial t} f\left(v_{i}^{2}, v_{1}, t\right)=\frac{8 \pi e^{2}}{m} \int d \vec{k} \mathscr{E}_{k} \frac{k_{1}^{2}}{k^{2}} \frac{\partial}{\partial v_{1}} \frac{1}{-i \omega+i k_{l} v_{1}} \frac{\partial}{\partial v_{1}} f\left(v_{\perp}^{2}, v_{1}, t\right)+\left(\frac{\partial f}{\partial t}\right)_{c}
$$

where $\perp$ and $\|$ refer to the direction of the magnetic field; $v$, the electron velocity; $k$, the wave number; $w$, the wave frequency; and $e$ and $m$ the charge and mass of the electron. The values of $\mathcal{E}_{\vec{k}}$, the spectral energy density, and $\mathcal{E}_{k_{1}}$, the parallel spectral energy density, are expressed in terms of the energy density, $W$, associated with the incident $r$ f-waves (with electric field amplitude E) by the relationship

$$
\int \mathrm{d} \vec{k} \mathcal{E}_{t}-\int \mathrm{dk}_{\mathrm{u}} \mathcal{E}_{\mathrm{k}_{\mathrm{l}}}=\boldsymbol{W}=\mathrm{E}^{\mathrm{T} / 8 \pi}
$$

The dispersion relation for lower hybrid waves, i.e.,

$$
\omega^{2}=\omega_{\mathrm{LH}}^{2}\left(1+\frac{k_{1}^{2}}{k^{2}} \frac{M}{m}\right) \quad \omega_{\mathrm{LH}}^{2}=\frac{\omega_{\mathrm{L} i}^{2}}{1+\omega_{\mathrm{pe}}^{2} / \omega_{\mathrm{ce}}^{2}}
$$

where $M$ is the ion mass and where $\omega_{p i}$. "wpe and $\omega_{c e}$ are the plasma ion, plasma eleatron and electron cyclotron frequencies, respectively, is used to eliminate $k_{\mathbb{l}} / k$ from the integrand in Eg. (A1). Therefore, the equation for the distribution function becomes

$$
\frac{\partial f}{\partial \tau}=\frac{\partial}{\partial H} D(H) \frac{\partial}{\partial W} f+\left(\frac{\partial f}{\partial \tau}\right)_{c}
$$


where we have expressed time in units of $v^{-1}$ defined by $v^{-1}=4 \pi n v v_{\mathrm{te}}^{3} / 3 \mathrm{k}^{4} \mathrm{pe}$ In $\mathrm{and}$ whore

$$
\begin{aligned}
& w=v_{\|} / v_{\text {te }} \\
& v_{t e}^{2}=k T_{e} / m \\
& D=\left.\frac{D_{0}}{w} \mathcal{E}_{k_{l}}\right|_{w / w v_{t e}} \\
& D_{0}=\frac{2 \pi \omega_{p i}^{2}\left(\omega^{2}-\hat{\omega}_{L H}^{2}\right)}{n m u v v_{t e}^{3} \omega_{L H}^{2}}
\end{aligned}
$$

In computing the collision term, we assume that the background distributions of both the electrons and ions are non-drifting, non-svolving Maxwellian distributions. Therefore, in the high velocity limit, valid for the resonant and nearby electrons, the collision term becomes

$$
\frac{\partial f}{\partial \tau}=\frac{z_{i}+1}{6 u^{3}} \frac{\partial}{\partial u}\left(1-\mu^{2}\right) \frac{\partial f}{\partial \mu}+\frac{1}{3 u^{2}} \frac{\partial}{\partial u}\left(-\frac{1}{u} \frac{\partial f}{\partial u}+f\right)
$$

where $u=v / v_{\text {te }}$ and $\mu=w / u$. It has been shown ${ }^{4}$ that the perpendicular velocity space dynamics play a minor role. Thus, we assume a Maxwellian perpendicular distribution and integrate over that direction ${ }^{9}$ so that $\mathrm{Eq}$. (A) 4 becomes

$$
\frac{\partial F(w)}{\partial \tau}=\frac{\partial}{\partial w} D(w) \frac{\partial}{\partial w} F(w)+\frac{2+Z i}{3} \frac{\partial}{\partial w}\left(\frac{1}{w^{3}} \frac{\partial}{\partial w}+\frac{1}{w^{2}}\right) F(w)
$$

where $F(w)=\int d \vec{v}_{1}$. When $z_{i}=1, E q$ ( (A7) reduces to Eq. (i) with $(\partial F / \partial T)_{c}$ given by Eq. (3).

The decay of $D(\omega)$ is governed by the equation

$$
\frac{\partial D}{\partial t}+v_{g x} \frac{\partial D}{\partial x}=2 \gamma^{\prime} D
$$


where the decay constant $\gamma^{*}$ and the group velocity $v_{\mathrm{gx}}$ for the lower hybrid electrostatic waves are

$$
\begin{aligned}
& \left.\gamma^{-}=\frac{\pi}{2} \frac{\omega_{\mathrm{Pe}}^{2}}{\omega} w \omega\right) \frac{k_{\|}^{2}}{k_{L}^{2}} \frac{\partial F}{\partial w}=\frac{\pi}{2} \frac{\omega_{\mathrm{Pe}}^{2}}{\omega} w|w|\left[\frac{\omega^{2}}{\omega_{\mathrm{LH}}^{2}}-7\right] \frac{m}{\mathrm{M}} \frac{\partial F}{\partial \omega}, \\
& v_{\mathrm{gK}}=U \mathrm{~W}, \quad \mathrm{U}=\frac{\omega_{\mathrm{LH}}^{2}}{\omega^{2}}\left[\frac{\omega^{2}}{\omega_{\mathrm{LH}}^{2}}-1\right]^{3 / 2}\left[\frac{\mathrm{m}}{\mathrm{M}}\right]^{1 / 2} v_{\mathrm{te}} .
\end{aligned}
$$

We express $t$ in units of $u^{-1}, v_{g x}$ in units of $u$ defined, in Eq. (A9), $x$ in units of $\mathrm{U} / \mathrm{u}$. Therefore $\mathrm{Eq}$. (A8) becomes

$$
\frac{\partial D}{\partial T}+v_{g} \frac{\partial D}{\partial \xi}=r D
$$

where

$$
v_{\mathrm{g}}=\mathrm{w}, \quad \xi=x u / U, \quad \gamma=2 v^{-1} \gamma^{-} .
$$

From Eq. (Ag) it follows that the wave damping rate can be written

$$
\gamma=A w|w| \frac{\partial F}{\partial w}, \quad \mathbf{A}=v^{-1} \pi \frac{\omega_{\mathbf{p i}}^{2}}{\omega}\left\{\frac{\omega^{2}}{\omega_{L H}^{2}}-1\right\} .
$$

When resonance broadening on $F$ is included. Eq. (A12) reduces to Eq. (4). 


\section{REFERENCES}

1. T. H. Stix, Phys. Rev. Lett. 15, 878 (1965).

2. N. J. Fisch, Phys. Rev, Lett. 41,873 (1978); Phys. Rev. Lett. 42 , 410 (1979).

3. T. H. Dupree, Phys. Fluids 2, 1773 (1966).

4. C. F. F. Karney and N. J. Fisch, Phys. Fluids 22, 1817 (1979).

5. N. J. Fisch and A. Bers, Phys. Rev, Lett. 35, 373 (1975).

6. N. J. Fisch, M,S, thesis, Massachusetts Institute of Technology, 1975.

7. R. C. Davidson, Methods in Nonlinear Plasma Theory, (Academic Press, New York, 1972), p. 166.

8. B. A. Trubnikov, in Reviews of Plasma Physics, edited by M. A. Leontovich (Consultants Bureau, New York, 1965), Vol. 1, p. 105.

9. A. A. Vedenov, in Review of Plasma Physics, edited by M. A. Leontovich (Consultants Bureau, New York, 1967), Vol. 3., p. 229. 


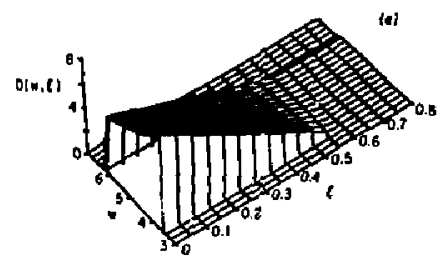

ig. 1. Wave power spectrum (in parallel velocity space) as a furiction of depth into the plasma. At the $\xi=0$, the power is zero except when $3.6<w<6.0$. In case (a) the effects of resonance broadening are omitted; in case (b) resonance broadening is included; and in case (c) resonance broadening is partially taken into account and thus treated inconsistently.
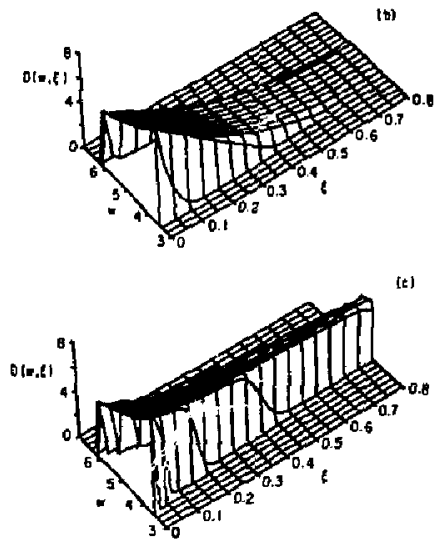

(PPPL-802146)

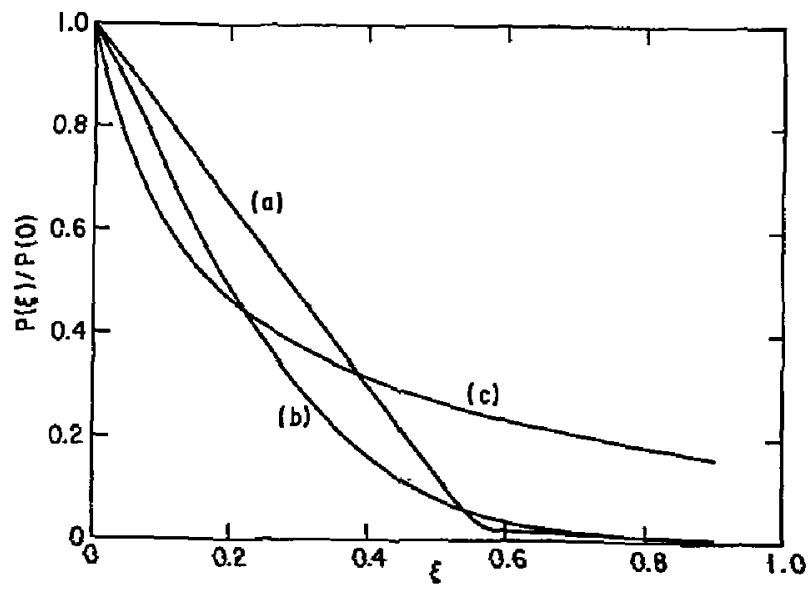

Fig. 2. Fraction of incident power retained by the $r f$ as a function of depth into the plasma- a) resonance broadening omitted, b) resonance broadening included, and c) resonance broadening partially taken into account. 

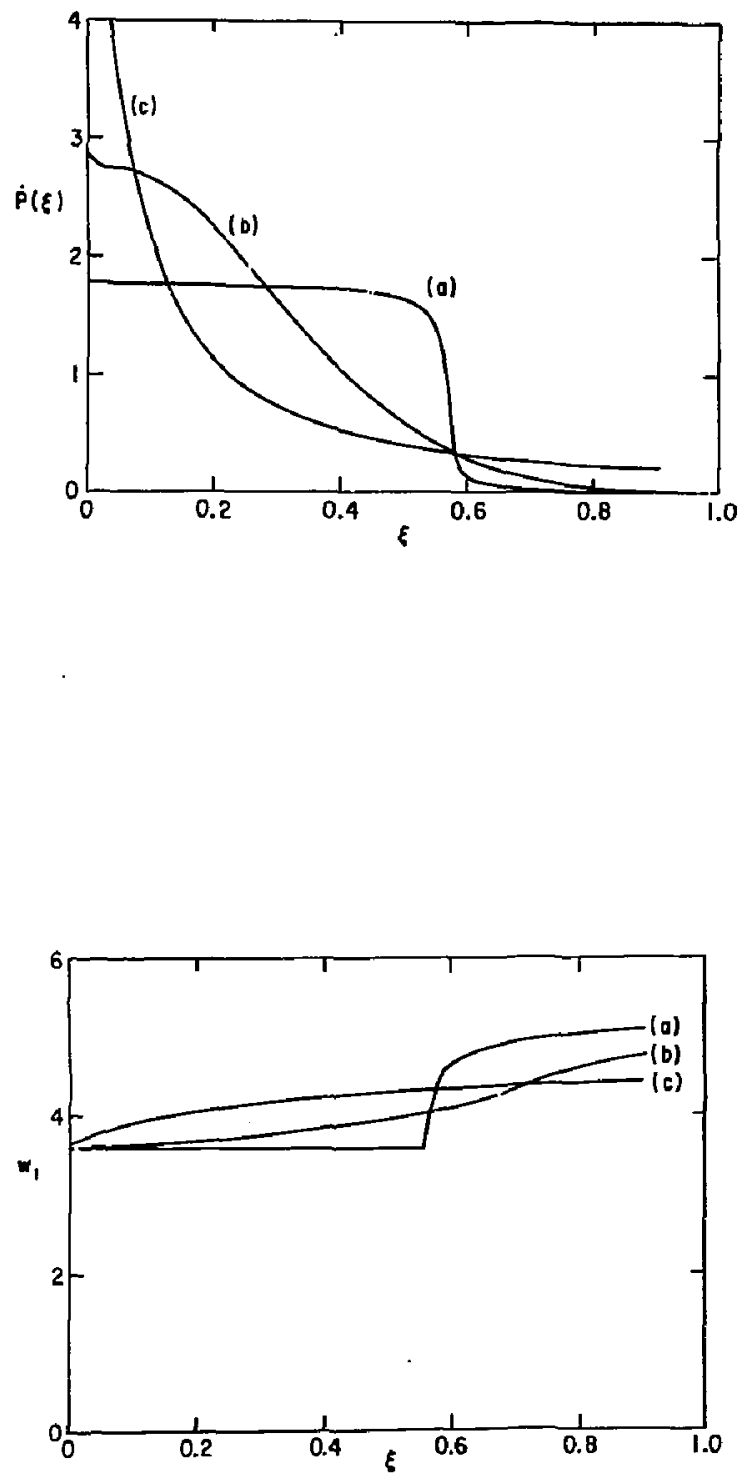

(PPPL-802148)

Fig. 3. Rate of rf-power deposition as function of depth into the plasma- a) resonance broadening onitted, b) resonance broadening included, and o) resonance broadening partially taken into account.

(PPPL-802147)

Fig. 4. Location of the inner (low velocity) edge of the rE-spectrum- a) resonance broadening cmitted, b) resonance broadening included, and c) resonance broadening partially taken into account. 